\title{
ADDED EFFECT OF PILATES MAT EXERCISES ON BALANCE AND LIMITS OF STABILITY IN CHRONIC STROKE PATIENTS: A PILOT STUDY
}

\author{
Prachi Sathe *1, Priya Chitre ${ }^{2}$, Snehal Ghodey ${ }^{3}$.
}

${ }^{* 1}$ BPTh Intern, MAEER's Physiotherapy College, Talegaon-Dabhade, India

${ }^{2}$ Associate Professor, MAEER's Physiotherapy College, Talegaon-Dabhade, India

${ }^{3}$ Principal, MAEER's Physiotherapy College, Talegaon-Dabhade, India

\section{ABSTRACT}

Background and Purpose: Stroke rehabilitation targets functional retraining of the limbs along with balance and gait. Even after extensive rehabilitation, up to $50 \%$ of stroke survivors experience motor deficits and balance problems that linger due to which the individuals are at high risk of falls. Trunk impairments though common do not manifest themselves as an individual problem and can impact balance. The purpose was to study the effect of Pilates mat exercises on balance and limits of stability in chronic stroke patients. It was hypothesized that since core training is not the point of focus in stroke rehabilitation, training the same through Pilates method could give better improvements in balance.

Subjects and Methods: 9 individuals were randomly allocated to either Pilates exercise group (PG, $n=5)$ or Control Group ( $C G, n=4)$.the $C G$ attended a regular rehabilitation program for stroke \& the balance exercises were progressed week-wise. The PG, in addition to conventional treatment, attended 18 Pilates exercise sessions over a period of 6 weeks ( 3 sessions / week). Tinetti Performance Oriented Mobility Assessment Scale (POMA) and Limits of Stability (LOS) including Reaction Time (RT), End Point Excursion (EPE), Maximum Excursion (MXE), Movement Velocity (MVL), and Directional Control (DC) were measured before and after the exercise program \& compared to assess the training effects. [Results]: Pilates exercises positively affected both dynamic balance and LOS in Chronic Stroke patients. For LOS, the RT (0.0427) and MXE (0.0292) components showed significant improvement in the PG while the improvements in CG were not significant. Other components of LOS-EPE, MVL, DC showed improvements clinically in both PG and CG but were not statistically significant via unpaired ' $\mathrm{t}$ ' test. For dynamic balance, the improvements in POMA score were more in PG than CG $(p=0.05)$ and were significant. Conclusion: This pilot study provides initial evidence that Pilates exercises may help improve balance and LOS in chronic stroke patients.

KEY WORDS: Balance, Limits of Stability, Pilates, Stroke.

Address for correspondence: Prachi Sathe, BPTh Intern, MAEER's Physiotherapy College, $2^{\text {nd }}$ floor, OPD complex, Bhausaheb Sardesai Talegaon Rural Hospital, Near Talegaon Railway station, Talegaon-Dabhade, Pune-410507, India. E-Mail: sathe_prachi19@rediffmail.com

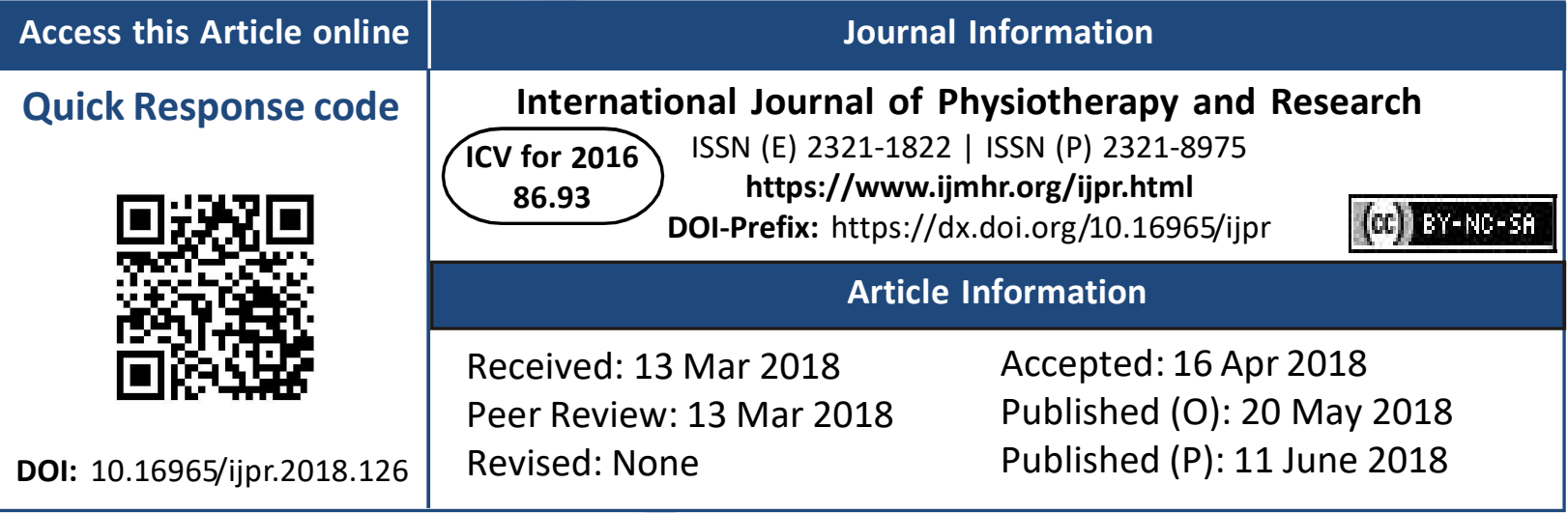

\section{INTRODUCTION}

Stroke, also called as cerebrovascular accident (CVA), is sudden loss of neurological function caused by an interruption of blood flow to the brain [1]. Motor deficits of hemiplegia, sensory dysfunction, balance impairments, communica- tion disorders, visual field defects, and cognitive impairment are the symptoms of CVA $[2,3]$. Balance impairment can be a direct effect of CVA as well as strength deficits post CVA. These deficits often lead to significant difficulty completing activities of daily living (ADLs). Even 
after extensive rehabilitation, up to $50 \%$ of stroke survivors experience lingering motor deficits and balance problems [3, 4].This balance impairment is the cause of high risk of falls in this population. It is reported that stroke patients are at high risk of falls with 1.3-6.5 falls/ person/year occurring and highest rates, 8.7falls/person/year, occurring after discharge from hospitals. Falls may lead to increased fear of falling, fractures which further cause activity restriction and hence depression [5]. Balance must therefore be improved in order to prevent falls and its consequences in this population.

Balance is a complex process involving interactions between the sensory and motor systems of the body. The visual, proprioceptive and vestibular system, neurological and musculoskeletal system and vertebrobasilar function along with the central functions of planning \& execution of movement are responsible for maintaining upright posture and balance $[2,6]$. Most of the rehabilitation in stroke patients revolves around functional retraining along with Balance and Gait. Strength deficits can be present in the extremities as well as the trunk. The strength deficits in trunk are perhaps not evident due to the bilateral cortical innervation of the same [2]. In post stroke hemiparesis patients, trunk performance is said to be an important independent predictor of ADLs after stroke; also trunk musculature weakness can affect balance, stability and functional abilities $[7,8]$.

Treatment to increase muscle activity has been a method to improve balance since diminished muscle activity has been identified as a contributing factor to balance impairment [3]. JungHyun Kim et. al. studied the effect of balance training on hemiplegic stroke patients and have stated that stroke impairs trunk control, which is required during weight shifting and equilibrium reactions [9]. Research in the field of postural control shows that sitting balance and extremity function require a certain level of trunk control and that loss of trunk strength occurs in all planes $[2,9]$. Training for trunk control and balance involves core stability exercises, strategy training, and weight shifts [1]. Also core stability results in proximal stability for distal mobility, a proximal to distal patterning of generation of force, and the creation of interactive movements of distal joints [2, 4].One of the components of static balance is the person's ability to maintain his/her center of gravity (COG) within the base of support (BOS). This can be measured by the Limits of Stability (LOS); it is the farthest distance in any direction a person can lean (away from midline) without altering the original base of support by stepping, reaching, or falling [2].It can be objectively measured using computerized dynamic posturography (NeuroCom ${ }^{\circledR}$ Basic Balance Master ${ }^{\circledR}$ System) $[6,10]$.

Literature has shown evidence that training the core muscles has shown improvements in functional capabilities as well. Researchers studying core training in stroke patients gave interventions such as core stabilization exercises with real time feedback, Tai Chi Chuan, Pilates, Proprioceptive Neuromuscular Facilitation (PNF), plank exercises $[6,11,3,13,12]$, of which Pilates became a target of interest as a useful exercise. With conventional treatment for stroke, core muscle training is not the point of focus. PILATES is one technique which is recently gaining more popularity. It helps in strengthening and conditioning of the body. It is a safe technique in which exercises are performed over a stable surface-the mat. Pilates exercises were developed by Joseph H. Pilates (1880-1967) as an exercise method to relax and strengthen the body[3].This technique aims to improve flexibility [14], core stability and balance by strengthening the core muscles of the body-Diaphragm, Transversus Abdominis (Tr.A), Multifidus, Pelvic floor [3].Presently there is availability of studies which show the effect of Pilates exercise on balance in normal, elderly individuals [15], individuals with multiple sclerosis $[16,17]$ and Parkinson's disease [18], however, the effects of same in stroke patients is limited. Therefore, the purpose of this study was to investigate the effects of 6 week of Pilates training on balance ability and LOS in chronic stroke patients.

\section{SUBJECTS AND METHODS}

Design: A randomized non-blinded controlled trial was chosen as the study. The study was performed in BSTRH Neurophysiotherapy OPD.

Subjects: Ambulatory subjects both male and 
female with chronic stroke ( 6 months post stroke and POMA score 19-24) having intact cognitive function (MMSE score 24-30) were chosen for the study. Individuals were excluded from the study if they had visual or hearing impairments; or contractures, tightness, deformities of the lower extremity; or lower motor neuron lesion of the lower extremity.

Assessments: Assessments were performed before and after exercise interventions. Dynamic balance assessment was performed using Tinetti Performance Oriented Mobility Assessment (POMA) scale. LOS assessment was done with the help of NeuroCom ${ }^{\circledR}$ Basic Balance Master $^{\circledR}$ as shown in Figure 1.

Interventions: After assessment all the subjects were divided into Group A: Experimental Group and Group B: Control Group. Group A was given functional rehabilitation according to their impairments for stroke, tailor-made conventional balance exercises and Pilates exercises. Group $B$ was given functional rehabilitation according to their impairments and tailor-made conventional balance exercises.
Conventional Balance Exercise Program: Exercise protocol was decided according to the ability of the patient as seen from the evaluation and the exercises were included accordingly in the program. Table 1 shows exercises which were included in Conventional Balance Exercise Program given to both the groups.

Pilates exercise program: In addition to functional rehabilitation and conventional balance exercises Group A was given Pilates exercises. Table 2 shows the exercises included in the Pilates exercise program. All the exercises were given to all patients, progressed week-wise for up to 6 weeks, no exercise discontinued, modified if required.

Statistical Analysis: Nine of ten subjects completed the study successfully. After 6 weeks of intervention, recorded data analyzed and compared using paired ' $\mathrm{t}$ ' test for matched data and unpaired ' $t$ ' test for comparing the two groups in the InStat software

Fig. 1: LOS Assessment using Balance Master.

'Patient file' is created recording their diagnosis, height, age for comparison with normative values after obtaining data

Patient made to stand bare foot on force plate with heel behind the horizontal black line, medial malleolus above the horizontal black line, lateral border of calcaneus in alignment with either the ' $S$ ', ' $M$ ' or ' $T$ ' vertical lines according to the height of the participant in a comfortable position, hands by the side palms facing the thigh

Cursor selected so as to ensure appropriate visual feedback. Participant shown the computer screen as above and asked to perform weight shifts so as to understand the real time feedback from the force plate

Participant instructed to move from the centre to the respective 8 directions one at a time after a green line appears at the bottom of the screen so as to measure reaction time

3 trials performed in each direction so as to minimize error 
Table 1: Exercises in Conventional Balance Treatment Program.

\begin{tabular}{|c|c|c|c|c|}
\hline Sitting legs uncrossed & \multicolumn{2}{|c|}{$\begin{array}{l}\text { Stepping forward, backward, } \\
\text { sideways }\end{array}$} & \multicolumn{2}{|c|}{$\begin{array}{c}\text { Foot on ball, moving ball sitting then } \\
\text { standing }\end{array}$} \\
\hline Sitting legs crossed & \multicolumn{2}{|c|}{ Marching in place } & \multicolumn{2}{|c|}{$\begin{array}{c}\text { Head rotation look up \& down sitting } \\
\text { then standing then walking }\end{array}$} \\
\hline Standing normal BOS (EO, EC) & \multicolumn{2}{|c|}{ Reach outs sitting and standing } & \multicolumn{2}{|c|}{$\begin{array}{c}\text { Trunk rotations sideways sitting then } \\
\text { standing then walking }\end{array}$} \\
\hline Tandem stance (EO, & \multicolumn{2}{|c|}{$\begin{array}{c}\text { Picking up objects off table, stool, } \\
\text { floor }\end{array}$} & \multicolumn{2}{|c|}{ Walking forward backward sideways } \\
\hline One leg stand (EO, EC) & \multicolumn{2}{|c|}{ Standing on foam (EO, EC) } & \multicolumn{2}{|c|}{$\begin{array}{c}\text { Dual task training (standing while } \\
\text { holding a ball over a book, standing } \\
\text { while catching a ball }\end{array}$} \\
\hline \multirow{5}{*}{$\begin{array}{l}\text { Table 2: Week- wise Exercise } \\
\text { Program for PILATES. }\end{array}$} & Week $1 \& 2$ & \multicolumn{2}{|c|}{ Week $3 \& 4$} & Week $5 \& 6$ \\
\hline & Ab Prep & \multicolumn{2}{|c|}{ Oblique Roll Back } & One leg circle \\
\hline & Breast Stroke Prep & \multicolumn{2}{|c|}{ Spine twist } & Obliques \\
\hline & Spine Stretch Forward & \multicolumn{2}{|c|}{ Saw } & Figure of eight \\
\hline & Half Roll Back & \multicolumn{2}{|c|}{ Hip Clamp } & Swimming Prep \\
\hline
\end{tabular}

\section{RESULTS}

Tables 3 and 4 provide data analysis with the resultant " $p$ " value.

Table 3: Data Analysis- Pre \& Post Values.

\begin{tabular}{|c|c|c|c|c|c|c|}
\hline \multirow{2}{*}{ Outcome Measures } & \multicolumn{2}{|c|}{ Group A: Experimental Group } & \multicolumn{2}{c|}{ Group B: Control Group } \\
\cline { 2 - 7 } & Pre & Post & “p” Value & Pre & Post & "p" Value \\
\hline Reaction Time (sec) & 1.592 & 1.182 & 0.0605 & 0.905 & 0.825 & 0.6603 \\
\hline End Point Excursion (\%) & 46.6 & 51.8 & 0.34669 & 58 & 61.25 & 0.424 \\
\hline Maximum Excursion (\%) & 59.2 & 69 & 0.0295 & 74.5 & 74.25 & 0.9448 \\
\hline Movement Velocity (degree/sec) & 2.58 & 2.66 & 0.8246 & 3.025 & 2.85 & 0.7423 \\
\hline Directional Control (\%) & 54.06 & 60.2 & 0.202 & 60.25 & 656 & 0.4534 \\
\hline POMA (points) & 20.2 & 23.4 & 0.0054 & 20.75 & 22.5 & 0.0354 \\
\hline
\end{tabular}

Table 4: Data Analysis between Groups.

\section{DISCUSSION}

\begin{tabular}{|c|}
\hline Outcome Measures \\
\hline Reaction Time (sec) \\
\hline End Point Excursion (\%) \\
\hline Maximum Excursion (\%) \\
\hline $\begin{array}{c}\text { Movement Velocity } \\
\text { (degree/sec) }\end{array}$ \\
\hline Directional Control (\%) \\
\hline POMA (points) \\
\hline
\end{tabular}

The purpose of this study was to determine effect of PILATES Mat Exercises on dynamic balance using POMA and LOS which includes Reaction Time (RT), End Point Excursion (EPE), Maximum Excursion (MXE), Movement Velocity (MVL) and Directional Control (DC) in chronic stroke patients. 10 subjects were assessed, 9 completed the study; out of these 5 underwent

\begin{tabular}{|c|c|c|c|}
\hline \multicolumn{2}{|c|}{ Mean Differences } & \multirow{2}{*}{ 'p' Value } & \multirow{2}{*}{ Significance } \\
\hline Group A & Group B & & \\
\hline-0.41 & -0.0325 & 0.0427 & Significant \\
\hline 5.2 & 3.25 & 0.3837 & Not Significant \\
\hline 9.8 & -0.25 & 0.0292 & Significant \\
\hline 0.08 & 0.175 & 0.335 & Not Significant \\
\hline 6.14 & 4.75 & 0.4208 & Not Significant \\
\hline 3.2 & 1.75 & 0.05 & Significant \\
\hline
\end{tabular}

Pilates mat exercise protocol along with conventional treatment for 6 weeks thrice a week. The other 4 participants were given only conventional treatment for the same duration.

Graph 1 and Table 3 we see that the RT reduced by $0.41 \mathrm{~s}$ in Group A whereas it reduced by $0.0325 \mathrm{~s}$ in Group B which means that the change is more in Group A with $p=0.0427$ which is statistically significant. Gonul Babayigit Irez et. al. 
Graph 1: Shows Mean differences of Reaction Time in the two groups.

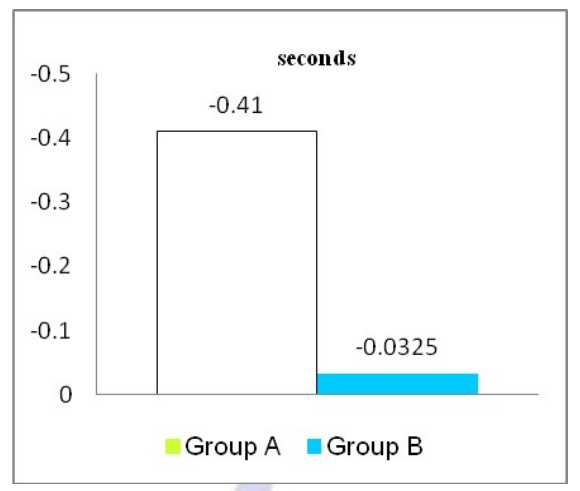

Graph 2: Shows Mean differences in End Point Excursion in the two Groups.

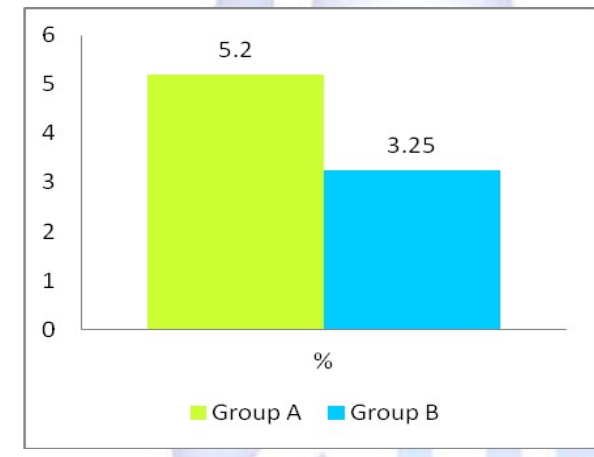

in their study on elderly women more than 65 years of age found that Pilates exercise improves RT [15]; however no direct correlation was found. It has been found in previous studies that exercises positively affect reaction time, but the effect of Pilates exercise on RT has not separately been studied. Yoga has similarities with Pilates exercises [15]; studies $[19,20]$ have shown that yoga improves reaction time by enhancing sensory-motor conduction velocity $\&$ the processing ability of the central nervous system. This has physiological as well as clinical significance as the persons with stroke will be able to react faster to any external stimuli from the environment which may be a causative factor to their loss of balance and result in a fall. It can also improve their concentration power and reduce mental fatigability during exercise training for better performance. Malathi and Parulkar in their study on RT have stated that improved RT could also be due to improved muscle tension/tone. They have also stated that RT is shown to be facilitated by a reasonable degree of initial muscle tension [21]. Improvement in RT suggests an improved capacity to respond to alterations in the COG [18].
Graph 3: Shows Mean differences in Maximum Excursion in the two Groups.

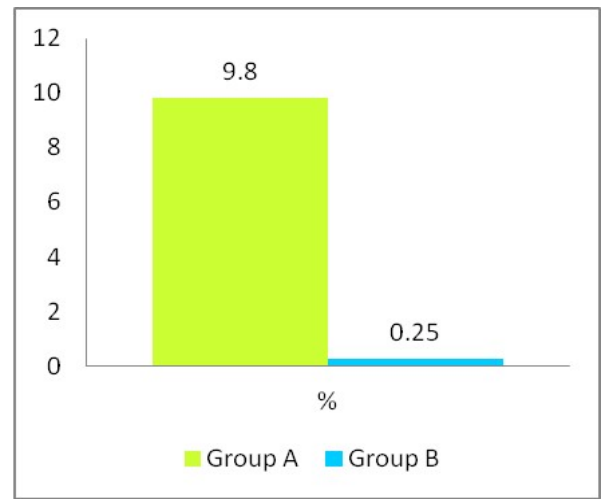

Graph 4: Shows Mean differences in Movement Velocity in the two Groups.

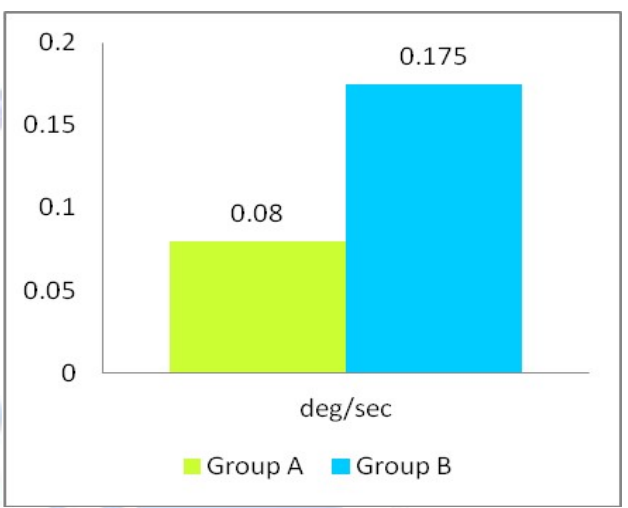

Graph 5: Shows Mean differences in Directional Control in the two Groups.

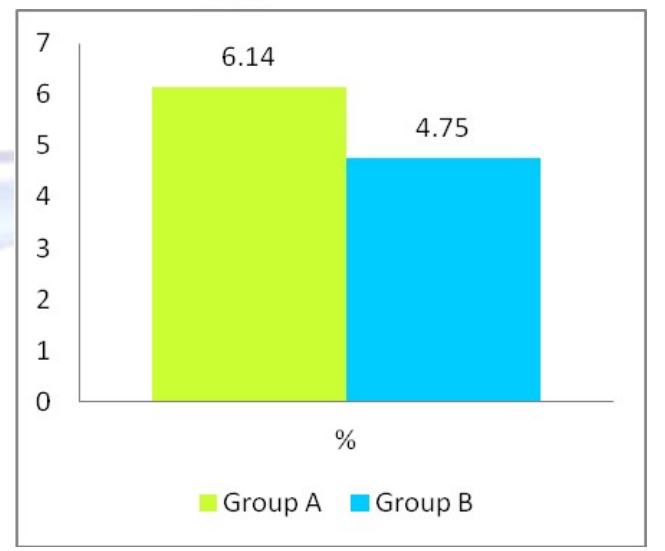

From Graph 3 and Table 4 it is seen that the MXE improved by $9.8 \%$ in Group A and reduced by $0.25 \%$ in Group B. The comparison between the two groups showed from Graph 3 Table 3 that there was statistically significant improvement with $p=0.0292$. With movement in any of the 8 directions, the opposite muscle group acts in an eccentric manner so as to control the movement in that direction which requires a good agonist-antagonist co-contraction of the trunk muscles [22]. With Pilates exercises there occurs training of the core muscles which helps improve trunk control. Trunk control allows the 
body to maintain the erect posture, to shift weight over the two limbs, to control all anti-gravity movements, to change body position in a controlled manner for balance and function [2]. Thus with Pilates exercise training, the same core and/or trunk muscles are targeted, and there occurs improvement in the strength of these muscles, thus improving the relation between the agonist and antagonist which helps for a better control of the joints for balanced movement [22]. The trunk muscles are targeted through Pilates exercises such as ' $A b$ prep', 'Breast Stroke prep', 'Spine Stretch forward', 'Half roll back' which help to achieve and maintain eccentric control. These changes can also be attributed to an additive effect of exercises from the conventional protocol such as picking up objects, reach-outs.

Graph 6: Shows Mean differences in POMA Scores in the two Groups.

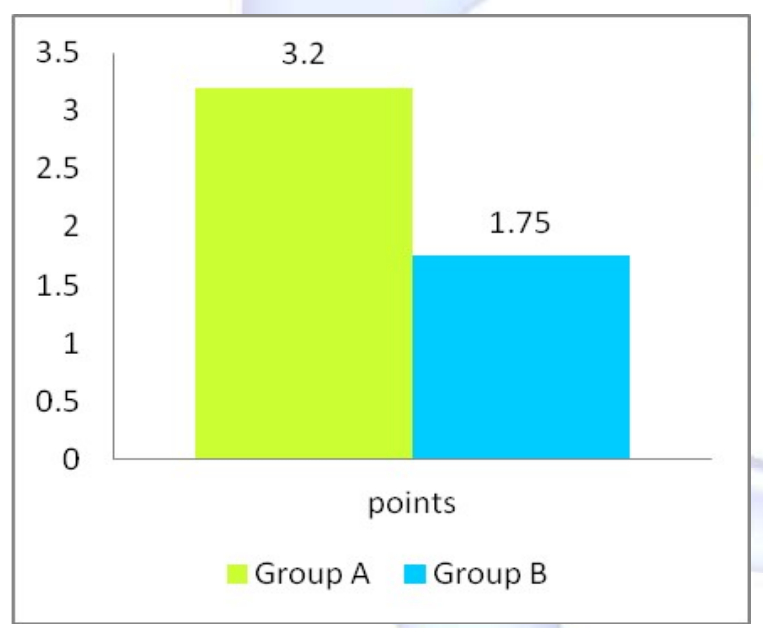

From Graph 6 and Table 4 it can be seen that, the POMA scores improved 3.2 points in Group $A$ and in Group $B$ it improved by 1.75 points. Pre and Post changes in the POMA scores in both the groups were statistically significant with $p=0.0054$ for Group A and $p=0.0354$ for Group $B$. When changes in the two groups were compared, from Graph 6 and Table 3 it was found that the change in Group A was more than that in Group B with $p=0.05$ which was statistically significant. The changes in the various components of POMA could be due to Pilates exercises since the exercises included in the Pilates protocol have similar muscle work as the components assessed with POMA. Conventional treatment includes functional exercises for improving balance hence the improvement in the POMA scores in Group B. However, the change in this group is less significant as compared to the experimental group. Conventional balance exercises are known to improve balance, however with Pilates exercise protocol, core muscles are engaged in performance of exercises. Core stability training regimens improve muscle activation patterns of the trunk [23]. With the 'imprint and release' exercise there is activation of Tr.A which can be performed during functional activities such as sit to stand to maintain balance [14].

Improvements in narrow BOS stance could be attributed to better control of COG even with a smaller BOS. Small amount of spontaneous postural sway is always present during quiet stance. Factors contributing to stability during quiet stance-alignment of the body, background muscle tone, postural tone [24]. Individuals with stroke demonstrate an increase in postural sway during standing [1]; it has been shown in previous studies that with Pilates exercises there is reduction in range and sway velocity of the center of gravity in chronic stroke patients which ultimately helps improve static balance [3]. To prevent loss of balance and falls due to an external perturbation, there occurs activation of ankle and hip strategies, depending on the magnitude of the perturbation [24]. Ankle strategy usually the first to get recruited, and is done in the distal-to-proximal direction by activation of muscles on the anterior side of body (ankle dorsiflexors'! quadriceps'! abdominals) followed by activation of muscles on posterior side (ankle plantar flexors '! hamstrings '! paraspinal muscles). With Pilates there occurs training of both abdominals and paraspinals which help to control the body better after an external perturbation. Core muscle strengthening through Pilates helps to improve and maintain proximal stability which helps to free a limb for mobility such as when turning around or during ambulation $[2,23]$. The 'hip release', 'hip clamp', 'one leg circle', 'swimming' exercises trains to move the lower limbs by maintaining the stable neutral position of the pelvis by targeting oblique muscles bilateral isometric action, multifidus, erector spinae muscles [12].

Improvements in gait can be attributed to improved internal and external oblique muscle strength. Reduced base of walking could be due 
to improved perception of balance, and better COG control. It could be because pilates exercise developed the deep muscles, such as the Tr.A, rectus abdominis and obliques [23] that take charge of the stability of the body and which may have helped to improve the stability of spine and the muscular strength and flexibility of the pelvis and hip joints [25]. The improved step symmetry and step continuity during walking maybe due to better recruitment of external and internal oblique muscles during gait [25]. 'Obliques roll back', 'spine twist', 'saw', 'obliques' exercises target the contralateral internal and external oblique muscles [26] along with predominantly active Tr.A during exercise which can be transferred to functional gait training for step symmetry and continuity. This also makes the gait symmetrical and reduces energy consumption during gait [9].

From Table 4, Graph 2, Graph 4 and Graph 5 it is seen that there were appreciable improvements in EPE, MVL and DC but these were not statistically significant. With core training there occurs improvement in proximal stability [2] with reduction in COP sway velocity [3] for a better mobility in the intended direction as is required when assessment of LOS. The improved DC could be attributed to improved proprioception of the trunk and lower extremities trained with conventional exercises and/or improved posture after Pilates training which may be due to increased muscle resistance and/or strength. However this improvement is time-dependent and involves adaptation of structures and postural awareness [22].

Thus this technique may help improve balance ability. However the effect of the same on risk of falls cannot be commented upon since the outcome measures did not take the same into consideration.

\section{CONCLUSION AND CLINICAL SIGNIFICANCE}

This pilot study concluded that PILATES Mat Exercises as a clinical intervention along with conventional treatment for chronic stroke patients could be more effective for improving Balance ability and Limits of Stability in this population.

This intervention can be easily applied in the clinical setting and as no additional equipment is required it is cost effective. Also, the exercises can be easily administered without the therapist requiring giving personal attention once the patient learns the exercise.

\section{ABBREVIATIONS}

CVA - Cerebrovascular Accident

ADLs - Activities of Daily Living

COG - Center of Gravity

BOS - Base of Support

LOS - Limits of Stability

POMA - Performance Oriented Mobility Assessment

RT - Reaction Time

EPE - End Point Excursion

MXE - Maximum Excursion

MVL-Movement Velocity

DC- Directional Control

Tr.A - Transverse Abdominis

Conflicts of interest: None

\section{REFERENCES}

[1]. O'Sullivan S, Schmitz T. Physical rehabilitation. 5th Ed.; 2007; p705, 721-722, 733, 754-755.

[2]. Umphred D. Umphred's Neurological Rehabilitation. 6th ed. St. Louis, Mo.: Elsevier/Mosby; 2013; p654, 658-659, 664, 686, 711, 732.

[3]. Lim H, Kim Y, Lee S. The effects of Pilates exercise training on static and dynamic balance in chronic stroke patients: a randomized controlled trial. Journal of Physical Therapy Science. 2016;28(6):18191824.

[4]. Chen I, Cheng P, Chen C, Chen S, Chung C, Yeh T. Effects of Balance Training on Hemiplegic Stroke Patients. Chang Gung Medical Journal. 2002;25(9):583-590.

[5]. Simpson L, Miller W, Eng J. Effect of Stroke on Fall Rate, Location and Predictors: A Prospective Comparison of Older Adults with and without Stroke. PLoS ONE. 2011;6(4):e19431.

[6]. Li F. The effects of Tai Ji Quan training on limits of stability in older adults. Clinical Interventions in Aging. 2014;:1261.

[7]. Karatas M, Çetin N, Bayramoglu M, Dilek A. Trunk Muscle Strength in Relation to Balance and Functional Disability in Unihemispheric Stroke Patients. American Journal of Physical Medicine \& Rehabilitation. 2004;83(2):81-87.

[8]. Verheyden G, Nieuwboer A, De Wit L, Feys H, Schuback B, Baert I, Jenni W, Schupp W, Thijs V, De Weerdt W. Trunk performance after stroke: an eye catching predictor of functional outcome. Journal of Neurology, Neurosurgery \& Psychiatry. 2006;78(7):694-698.

[9]. Kim J, Lee S, Jeon S. Correlations among trunk impairment, functional performance, and muscle activity during forward reaching tasks in patients with chronic stroke. Journal of Physical Therapy Science. 2015;27(9):2955-2958. 
[10]. Natus Medical Incorporated - NeuroCom Balance Master Systems [Internet]. Natus.com. 2018 [cited 2018 Jan 3]. Available from: http://www.natus.com/ index.cfm?page=products_1\&crid=271

[11]. Chang Y, Nien Y, Tsai C, Etnier J. Physical Activity and Cognition in Older Adults: The Potential of Tai Chi Chuan. Journal of Aging and Physical Activity. 2010;18(4):451-472.

[12]. Ekstrom R, Donatelli R, Carp K. Electromyographic Analysis of Core Trunk, Hip, and Thigh Muscles During 9 Rehabilitation Exercises. Journal of Orthopedic \& Sports Physical Therapy. 2007;37(12):754-762.

[13]. Mesquita L, de Carvalho F, Freire L, Neto O, Zângaro R. Effects of two exercise protocols on postural balance of elderly women: a randomized controlled trial. BMC Geriatrics. 2015;15(1).

[14]. Phrompaet S, Paungmali A, Pirunsan U, Sitilertpisan P. Effects of Pilates Training on Lumbo-Pelvic Stability and Flexibility. Asian Journal of Sports Medicine. 2011;2(1).

[15]. Babayigit Irez G, Ali Ozdemir R, Evin R, Gokhan Irez $\mathrm{S}$, Korkusuz F. Integrating Pilates exercise into an exercise program for $65+$ year-old women to reduce falls. Journal of Sports Science and Medicine. 2011;(10):105-111.

[16]. Mohammad Marandi S, Shayegan Nejad V, Shanazari Z, Zolaktaf V. A Comparison of 12 Weeks of Pilates and Aquatic Training on the Dynamic Balance of Women with Multiple Sclerosis. International Journal of Preventive Medicine. 2013;4(1):S110-7.

[17]. Soysal Tomruk M, Uz M, Kara B, Ýdiman E. Effects of Pilates exercises on sensory interaction, postural control and fatigue in patients with multiple sclerosis. Multiple Sclerosis and Related Disorders. 2016;7:70-73.

[18]. Johnson L, Putrino D, James I, Rodrigues J, Stell R, Thickbroom G, Mastaglia F. The effects of a supervised Pilates training program on balance in Parkinson's disease. Advances in Parkinson's disease. 2013;02(02):58-61.
[19].MADANMOHAN, THOMBRE D, BALAKUMAR B, NAMBINARA YANAN T, THAKUR S, KRISHNAMURTHY $\mathrm{N}$, CHANDRABOSE A. EFFECT OF YOGA TRAINING ON REACTION TIME, RESPIRATORY ENDURANCE AND MUSCLE STRENGTH. Indian J Physiol Pharmacol. 1992;36(4):229-233.

[20]. Bhavanani A, Sanjay Z, Madanmohan, Dayanidy G, Basavaraddi I. Effect of yoga therapy on reaction time, biochemical parameters and wellness score of peri and post-menopausal diabetic patients. International Journal of Yoga. 2012;5(1):10.

[21]. MALATHI A, G. PARULKAR V. EFFECT OF YOGASANAS ON THE VISUAL AND AUDITORY REACTION TIME. Ind J Physiol Pharmac. 1989;33(1):110-112.

[22]. Sinzato C, Taciro C, Pio C, Toledo A, Cardoso J, Carregaro R. Efeitos de 20 sessões do método Pilates no alinhamento postural e flexibilidade de mulheres jovens: estudo piloto. Fisioterapia e Pesquisa. 2013;20(2):143-150.

[23]. Kahle N, Gribble P. Core Stability Training in Dynamic Balance Testing Among Young, Healthy Adults. Athletic Training \& Sports Health Care. 2009;1(2):6573.

[24]. Shumway-Cook A, Woollacott M. Motor control: Translating Research Into Clinical Practice. 3rd ed. 2006; p160-164

[25]. Roh S, Gil H, Yoon S. Effects of 8 weeks of mat-based Pilates exercise on gait in chronic stroke patients. Journal of Physical Therapy Science. 2016;28(9):2615-2619.

[26]. Urquhart D, Hodges P. Differential activity of regions of transversus abdominis during trunk rotation. European Spine Journal. 2004;14(4):393-400.

\footnotetext{
How to cite this article:

Prachi Sathe, Priya Chitre, Snehal Ghodey. ADDED EFFECT OF PILATES MAT EXERCISES ON BALANCE AND LIMITS OF STABILITY IN CHRONIC STROKE PATIENTS-A PILOT STUDY. Int J Physiother Res 2018;6(3):2732-2739. DOI: 10.16965/ijpr.2018.126
} 